\title{
Alan Walker (ed.) (2018). The New Dynamics of Ageing. Volume 1. Great Britain: Policy Press, 368 pp. ISBN 9781447314738 (paperback) Alan Walker (ed.) (2018). The New Dynamics of Ageing. Volume 2. Great Britain: Policy Press, 331 pp. ISBN 9781447314790 (paperback)
}

\author{
Reviewed by ShyH PoH TeO*
}

The New Dynamics of Ageing (NDA) is a large-scale multidisciplinary research programme from the United Kingdom, funded through five research councils. The research projects span a wide range of aspects related to older people, including arts and humanities, biological sciences, engineering, social sciences and medical research. It was an ambitious goal of the editor to cover the scope of the NDA research into these two volumes. This was achieved through selecting key projects to illustrate the value of multidisciplinary collaboration and engagement of older people. The breadth of topics covered provides insight into considerations from other relevant disciplines, with a focus on practical implications that will benefit older individuals and society. These books are recommended for those involved in gerontology and geriatrics, and other specialties involved in service provision and policy planning for older people. While the books

*Shyh Poh Teo, Geriatrics and Palliative Unit, Department of Internal Medicine, RIPAS Hospital, Brunei Darussalam 
International Journal of Ageing and Later Life

do not exhaustively cover all the completed NDA research, a complete list of projects is available in each volume for reference.

The two volumes follow a consistent format, providing a useful background of the problem at hand, research methodology used, followed by key findings and discussion of implications. The projects are clustered into themes within the books, ranging from active and healthy ageing, design for an older population, biological perspectives of ageing and how older people are represented in art and media. This arrangement allows readers to delve into different sections, particularly those that are relevant to their professional interests, rather than reading the books from cover to cover.

The main strength of the book is the clear introduction and background information provided at the beginning of each chapter, putting the research into the context of current knowledge and understanding. As each project covers very specific topics, it is important that readers possess basic knowledge to understand the relevance and significance of the research findings. I found the chapter on biological drivers of cell ageing highly specialised, particularly descriptions of cell theory and senescence. However, the authors managed to break down and explain the basics well, before delving into the genetic and molecular techniques used in their study.

While large national research frameworks tend to be useful for the country of origin, the NDA programme covered a diverse range of settings, providing learning points that are generalisable and applicable to other localities. The section on community engagement covered urban activities involving art and music. This was followed by a chapter describing a project in rural areas, allowing readers to contrast the different considerations for connecting older people with meaningful activities. These multiple facets within each theme covered through connecting the different projects together provide a more complete and holistic picture that would not have been apparent through reading the individual chapters published for each project. There were also examples of international collaboration under the NDA, such as the population studies carried out in India, Brazil and South Africa. The comparative nature of these chapters demonstrates how changes in social policy have real impact on the well-being of older people. The authors managed to distil clearly the 
relevant real-life lessons from these countries, which are relevant to policy-makers internationally.

Another strength of the book is the summary of key findings and implications for practice at the end of each chapter. The conclusion at the end of each volume also reiterates learning points that require emphasis. All too often, research focuses on a very specific aspect of a topic, with limited practical value. In this case, the NDA researchers have packaged the information into usable, applicable evidence-based information that can be easily referred to.

There are two downsides to the way the books were arranged. I felt there was too much detail covering research methodology, particularly for the quantitative studies. While some readers may wish to critically appraise the study or plan to replicate the project, references were provided which they could look up if required. In addition, while there was a wide target audience for these texts, the detailed discussions within each project may be relevant to specific professionals only. For example, our physiotherapists may be interested in the chapter on dynamic biomechanical data and stair negotiation, but it may be difficult to generate their enthusiasm for reading other sections such as kitchen design or representations of older people in art. However, I would still utilise these two volumes as reference material, highlighting aspects that each speciality should focus on to allow them to expand their scope of practice.

Reading about the NDA projects was an eye-opener, as the breadth of research provided many ideas that can be considered or implemented locally. For example, I learned about the possibility of assessing the impact of government funding plans using projection models on costs of longterm care, such as in the Modelling Needs and Resources of Older People to 2030 (MAP2030) project, which would be useful for service planning.

The research outcomes also add to the existing evidence base that supports current action plans and initiatives for older people. Healthy ageing across the life course is a useful concept to maximise healthy years without disability in older people. The chapter on the Healthy Ageing across the Life Course (HALCyon) project describes cohort studies confirming the robust association between poor socio-economic circumstances in childhood and lower capabilities in later life, while genetic effects on physical and cognitive capabilities were limited. This resonated well with 
International Journal of Ageing and Later Life

our practice and should motivate health professionals to apply this approach of proactively caring for people's health at all ages due to longterm implications for future well-being.

The NDA framework also provided a crucial reminder that research for older people requires multidisciplinary input and their inclusion as active participants. The projects show that external views from different angles open up potential solutions and opportunities. For example, the collaborative Working Late project identified recommendations by older people to identify health needs and flexible working practices to support longer work hours. The process of involving older people in developing the Older People's Quality of Life Questionnaire also ensured their perspectives were taken into account and the tool was relevant. I thought the involvement of older people to design fit-for-purpose clothing and performance sportswear was quite inspired. It was also motivating to see action research with mutually beneficial outcomes, such as sustainable community groups, through the participatory action research process.

I enjoyed reading the quotes from the NDA qualitative research, which provided participants with a voice to share their views on community engagement, employment in later life, adjustments in a novel environment and what constitutes dignity and quality of life. The expression of older people in art serves as a reminder to clinicians regarding personal aspects such as self-image. The "representing self - representing ageing" project engaged older women in creating visual images, which was a novel approach to critique and challenge persistent media stereotypes that older people are dependent and unable to manage. The Ages and Stages project also showed how older people have a role in constructing individual and community identities through involvement in theatre and continued social engagement.

Overall, the book delivers useful insights into diverse aspects of older people's lives and reminds readers of the importance of involving older people and collaboration between multiple disciplines in research. I would recommend this book to healthcare professionals and policy-makers to aid the understanding of ageing and its implications. 